\title{
Cell-Line Annotation on Europe PubMed Central
}

\section{Contents}

1 Introduction . . . . . . . . . . . . . . . . 2

1.1 Why do we tag cell lines? . . . . . . . . . . . . . . 2

1.2 Linking Europe PMC articles to cell lines . . . . . . . . . . 2

2 A large-scale annotation and analysis pipeline . . . . . . . . 2

2.1 Dictionary builder ................. 3

2.2 Semantic tagging engine . . . . . . . . . . . . . . 4

2.3 Analysis and summary report generation module . . . . . . . . 4

3 Results........................... 4

3.1 Cellosaurus-based cell-line dictionary . . . . . . . . . . . . . 4

3.2 Annotation results on Open Access (OA) PMC collection . . . . 6

3.2.1 Annotation Example .............. 6

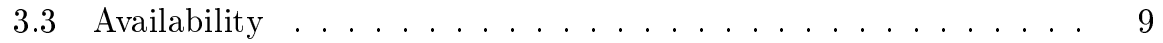

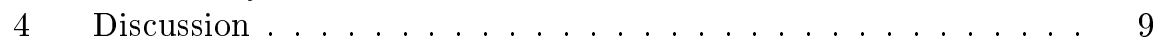

\section{List of Tables}

1 A mapping table between an ontology and a dictionary. . . . . . 4

2 About Cellosaurus-based (version 8.0) cell-line dictionary. . . . . 5

3 Top 30 most frequent terms (collection frequency). . . . . . . . . 7

4 Top 15 most frequent concept IDs. . . . . . . . . . . . . . 8

\section{List of Figures}

1 A pipeline for a large scale annotation and analysis given an ontology. . . . . . . . . . . . . . . . 3

2 Dictionary example...................... 5

3 Annotation example on an OA article (PMC1266034) . . . . . 6

\section{Abstract}

A cell line is a cell culture developed from a single cell and therefore consisting of cells with a uniform genetic make-up. A cell line has an important role as a research resource such as organisms, antibodies, constructs, knockdown reagents, etc. Unique 
identification of cell lines in the biomedical literature is important for the reproducibility of science. As data citation, resource citation is also important for resource re-use.

In this paper, we mention the challenges of identifying cell lines and describe a system for cell line annotation with preliminary results.

\section{Introduction}

\subsection{Why do we tag cell lines?}

A cell line is a cell culture developed from a single cell and therefore consisting of cells with a uniform genetic make-up. A cell line has an important role as a research resource such as organisms, antibodies, constructs, knockdown reagents, etc. Unique identification of cell lines in the biomedical literature is important for the reproducibility of science [7]. As data citation, resource citation is also important for resource re-use [1]. Identifying cell lines is a nontrivial problem with the following challenges and difficulties:

- A significant number of cell line names consist of only numbers.

- A significant number of cell line names consist of less than 4 letters.

- Cell line names often look similar with gene/protein names.

- Cell line names sometime look similar with person names.

\subsection{Linking Europe PMC articles to cell lines}

Europe PubMed Central is a database of life science research articles and abstracts, including PubMed (http://europepmc.org) [4]. One of main services on Europe PMC is to link full-text articles to biological data sets or databases by two methods:

- Named Entity Recognition

- Accession Number Mining [2]

Combined with other features on Europe PMC, cell line annotation can be useful. For example, give me all articles where cell line $\mathrm{X}$ is mentioned only in Methods section.

In this report, we describe our work on linking articles to research resources using our cell line tagger and section tagger.

\section{A large-scale annotation and analysis pipeline}

Recently, we have developed a system which can generate a dictionary from a given ontology or terminological resource, and performs a large scale analysis of dictionary usages. The system mainly consists of three modules: 1) dictionary building module, 2) semantic tagging module, and 3) analysis and report generation module. Figure 1 shows an diagram of the system architecture. 


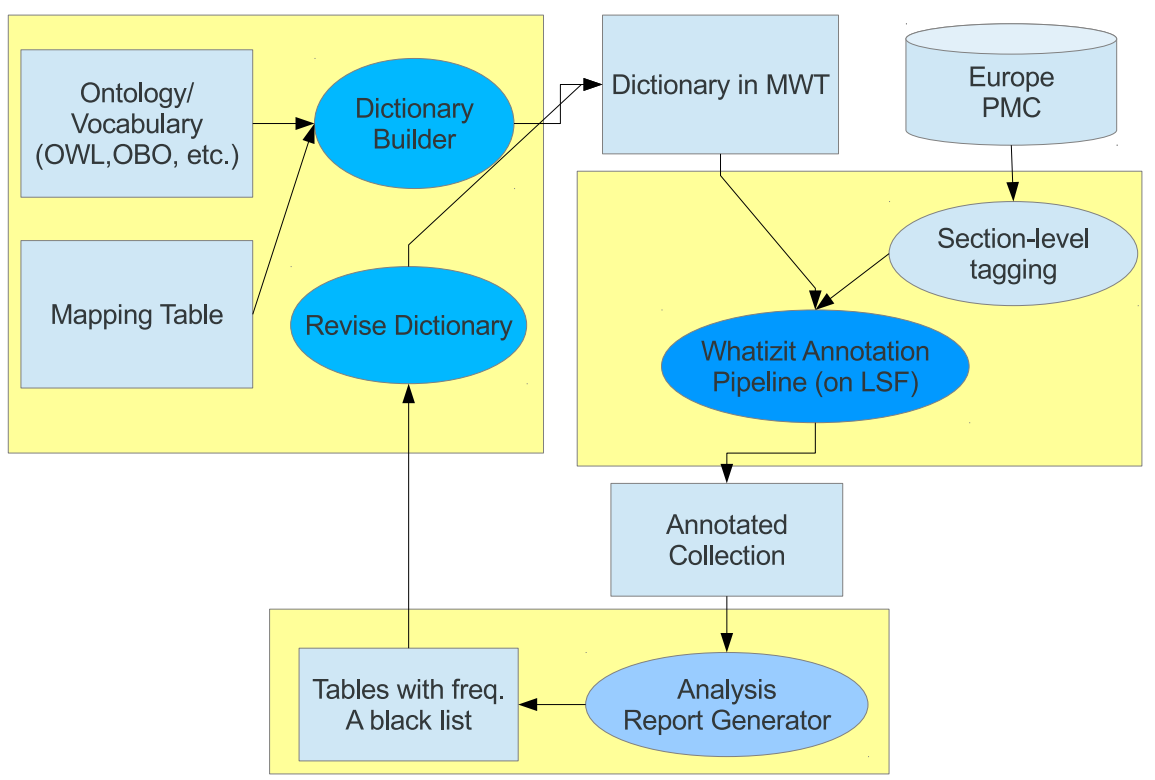

Fig. 1: A pipeline for a large scale annotation and analysis given an ontology.

\subsection{Dictionary builder}

Dictionary builder is a module that generates a dictionary (in mwt format used in Whatizit), given an ontology, controlled vocabulary, or any other terminological resources. A number of input formats supported are as follows:

- Web Ontology Language (OWL) Recently, there has been a trend to develop ontologies in OWL recommended by $\mathrm{W}_{3} \mathrm{C}^{1}$. This module can generate dictionaries from ontologies in Web Ontology Language (OWL) using SPARQL Query Language for RDF.

- Open Biomedical Ontologies (OBO)

- Swiss-Prot style format

Besides the given ontology, additional information is required for building a dictionary as follows:

- A mapping table between an ontology and a dictionary (as in Table 1), and is- $a$ relationship if any exists. 
Tab. 1: A mapping table between an ontology and a dictionary. In general, one concept ID has one or more than one terms.

\begin{tabular}{cc}
\hline Ontology (or Terminological Resource) & Dictionary \\
\hline Class, Attribute, or Field & Term \\
Class, Attribute, or Field & Concept ID \\
Class, Attribute, or Field & Attribute \\
\hline
\end{tabular}

- A list of filtering rules. For example,

- Remove a term of which the length is less than N.

- Remove a term that has only digits.

- A list of regular expressions (e.g., for accession numbers)

\subsection{Semantic tagging engine}

This module consists of a list of taggers based on java finite automata [5] and MALLET (MAchine Learning for LangugE Tookit ${ }^{2}$ ): Given a dictionary (generated in the previous step) in $\mathrm{mwt}^{3}$ format, this annotation pipeline can annotate documents in a dictionary-based approach. We can plug in different semantic taggers running on Whatizit server.

\subsection{Analysis and summary report generation module}

A large scale annotation analysis module based on Hadoop, Pig Latin (for counting), and $\mathrm{R}$ language (for visualization). One application of this analysis module is to help users with building a customized list of stop words and revising a dictionary based on summary report.

One method to evaluate the importance of a term is to us its frequency [3]. This frequency information can be used to find a list of stop words for domain-specific applications instead of using a default list of stop words.

\section{Results}

\subsection{Cellosaurus-based cell-line dictionary}

We have performed a preliminary analysis on Cellosarus and Cell Line Ontology (CLO). In this analysis, we have found that terms in Cellosarus are more often matched on free texts from biomedical corpora, suggesting the usage as a dictionary. On the other hand, CLO terms are more conceptual and less matched.

\footnotetext{
${ }^{1}$ http://www.w3.org/TR/owl-features/

2 http://mallet.cs.umass.edu/

3 http://monqjfa.berlios.de/monqApiDoc/monq/programs/DictFilter.html
} 
Tab. 2: About Cellosaurus-based (version 8.0) cell-line dictionary.

\begin{tabular}{crr}
\hline & Before filtering & After filtering \\
\hline \# Concepts & 23,115 & 23,107 \\
\# Terms & 39,089 & 39,042 \\
\hline
\end{tabular}

$<\mathrm{mwt}>$

$<$ cell_line ids="\%1" species=" $\% 2$ " type=" $\% 3 "$ sex=" $\% 4 "$ disease=" $\% 5 "$

crosslink $=" 1 \% 6 ">\% 0</$ cell_line $>$

<r p1="CVCL_E548" p2="Homo sapiens" p3="Transformed cell line" p4="Female" p5="unknown"

p6="unknown" $>\# 15310-\mathrm{LN}</ \mathrm{r}\rangle$

$\langle/$ mwt $>$

Fig. 2: Dictionary example

Based on this, we have chosen Cellosaurus which is a controlled vocabulary of cell lines developed by Swiss Institute of Bioinformatics (SIB).

With the dictionary building module (mentioned in Section 2.1), we generated a cell-line dictionary for named entity recognition as follows:

- In a mapping table, we mapped ID (IDentification) and SY (SYnonyms) fields to terms, and AC (ACcession number) field to concept IDs.

- Following is a list of filtering/transformation rules:

- Only terms with more than 3 letter terms are used to build dictionaries.

- Less than four letters + 'cell' (as the following constraint word)

- Only numbers + cell

- Following is a list of terms added into our blacklist, provided by domain experts:

- Cancer, Center, Grey, Spindle, Chance, Patches, Bones, Horse, TIME, Set-2, Renal carcinoma, Badger, Chew, Moose, Marry, Scout, COST, Pinwheel, Giant cell tumor, Fetch, Mint, CHOP, Ears, Jersey, Chase, Chief, Flip, Guard, Junior, Stripes, Squirrel, Typhoon, Sage, Had-1, Speedy, Thyme, WISH, Kin-, Tackle, Pepper, Taurus, WART, Speckles, Soccer, Buttons, Gemini, Bing (47 terms)

Table 2 shows statistics on the dictionary built by this module and Figure 2 shows an example of the dictionary generated based on these rules. 
Kinetics of <chemical>capsaicin</chemical>-activated currents in $<$ cell_line>HEK293</cell_line> cells stably expressing <organism>rat</organism> $<$ gene $>$ TRPV $1</$ gene $>$ channels .

Fig. 3: Annotation example on an $\mathrm{OA}$ article (PMC1266034)

\subsection{Annotation results on Open Access (OA) PMC collection}

For a large scale analysis, first we applied our section tagger to $633,174 \mathrm{OA}$ full-text articles from Europe PubMed Central collection, and annotated these section-labeled articles. The rational for using section-labeled articles is to find different usages of cell lines over different sections such as Introduction, Methods, Results, and Discussion [6]. Then, we applied our gene/protein name tagger in order to reduce confusions between gene/protein and cell line names.

After the annotation we performed three different analyses: concept-wise, term-wise, and article-wise in order to find different aspects of ontology usages.

In term-wise analysis, each term was counted separately. Table 3 shows the results. In this table, we can see section-specific false positive cases. For example, there are some false positive terms specific in Methods section such as Fisher and Madison. Different sections have different false positive categories suggesting needs for a section-dependent blacklist.

- Kobayashi in Introduction vs Kobayashi in Methods

- Promega Corporation, Madison, WI, USA.

Based on the results above, we removed term Focus, which is often used as "Focus group" in Methods sections.

HeLA was not the most frequent term in Methods section because of two false positive terms (i.e., Fisher and Madison).

Concept-wise analysis: All terms (synonyms, orthographic variants, etc) belonging to one concept were considered as the same. Table 4 shows 15 most frequent concepts.

\subsubsection{Annotation Example}

Figure 3 shows an excerpt of an annotated article on gene expression experiments. 
Tab. 3: Top 30 most frequent terms (collection frequency).

A number of chunks for each section is as follows: 57,291,268 for Introduction, 98,112,562 for Methods, 156,007,208 for Results, and 83,851,662 for Discussion.

\begin{tabular}{|c|c|c|c|c|c|c|c|}
\hline \multicolumn{2}{|c|}{ Introduction } & \multicolumn{2}{|c|}{ Methods } & \multicolumn{2}{|c|}{ Results } & \multicolumn{2}{|c|}{ Dicussion } \\
\hline HeLa & 3068 & Fisher & 28966 & HeLa & 52563 & HeLa & 7662 \\
\hline TLR4 & 1934 & Madison & 23793 & MCF-7 & 21262 & MCF-7 & 5984 \\
\hline MCF-7 & 1778 & HeLa & 21689 & HEK293 & 17357 & TLR4 & 4173 \\
\hline Fisher & 1499 & $293 \mathrm{~T}$ & 9073 & HepG2 & 14381 & HepG2 & 3695 \\
\hline TLR2 & 1353 & HEK293 & 8671 & $293 \mathrm{~T}$ & 12425 & TLR2 & 3380 \\
\hline HepG2 & 1218 & MCF-7 & 6663 & HCT116 & 11396 & $\mathrm{PC} 12$ & 3200 \\
\hline $\mathrm{PC} 12$ & 1205 & BL21 & 5722 & MCF7 & 10412 & $\mathrm{LNCaP}$ & 2499 \\
\hline Jensen & 1191 & HepG2 & 5299 & LNCaP & 9574 & MDCK & 2341 \\
\hline Hughes & 1156 & Vero & 5202 & $\mathrm{PC} 12$ & 8082 & HEK293 & 2315 \\
\hline Murphy & 1077 & MDCK & 4515 & MDCK & 7872 & MCF7 & 2044 \\
\hline Becker & 841 & HEK293T & 4497 & $\mathrm{C} 2 \mathrm{C} 12$ & 7622 & 3T3-L1 & 1773 \\
\hline Kobayashi & 739 & Focus & 3981 & HEK293T & 7324 & $\mathrm{C} 2 \mathrm{C} 12$ & 1701 \\
\hline LNCaP & 737 & HCT116 & 3246 & $\mathrm{U} 2 \mathrm{OS}$ & 6589 & $\mathrm{PC}-3$ & 1682 \\
\hline Cole & 710 & MCF7 & 3000 & Fisher & 6543 & HCT116 & 1653 \\
\hline HEK293 & 703 & $\mathrm{C} 2 \mathrm{C} 12$ & 2976 & SH-SY5Y & 6393 & Fisher & 1481 \\
\hline LC-MS & 681 & $\mathrm{PC} 12$ & 2923 & TLR4 & 5738 & SH-SY5Y & 1391 \\
\hline $\mathrm{C} 2 \mathrm{C} 12$ & 670 & LNCaP & 2721 & $\mathrm{PC}-3$ & 5408 & Jensen & 1306 \\
\hline MDCK & 668 & LC-MS & 2710 & Vero & 5222 & SP cells & 1245 \\
\hline 3T3-L1 & 663 & Fuji & 2657 & NIH3T3 & 5201 & HT-29 & 1169 \\
\hline Fang & 661 & COS-7 & 2495 & TLR2 & 4877 & Murphy & 1164 \\
\hline Vogel & 570 & SH-SY5Y & 2434 & 3T3-L1 & 4642 & Hughes & 1077 \\
\hline MCF7 & 540 & $\mathrm{~F} 4 / 80$ & 2431 & $\mathrm{HaCaT}$ & 4498 & NIH3T3 & 1026 \\
\hline Otto & 499 & $\mathrm{U} 2 \mathrm{OS}$ & 2251 & COS-7 & 4376 & HТ29 & 1024 \\
\hline $\mathrm{PC}-3$ & 488 & NIH3T3 & 2246 & SW 480 & 4333 & Vero & 1016 \\
\hline SP cells & 479 & 293 cells & 2207 & НТ29 & 4320 & $\mathrm{HaCaT}$ & 972 \\
\hline Peer & 473 & 3T3-L1 & 1919 & DU 145 & 4197 & Kobayashi & 943 \\
\hline Focus & 469 & HEK 293 & 1849 & RAW264.7 & 3846 & DU145 & 932 \\
\hline SH-SY5Y & 463 & RAW 264.7 & 1844 & HT-29 & 3759 & $293 \mathrm{~T}$ & 883 \\
\hline DT40 & 452 & RAW264.7 & 1813 & H1299 & 3676 & RAW264.7 & 847 \\
\hline HCT116 & 443 & HEK-293 & 1790 & T47D & 3661 & Becker & 804 \\
\hline
\end{tabular}




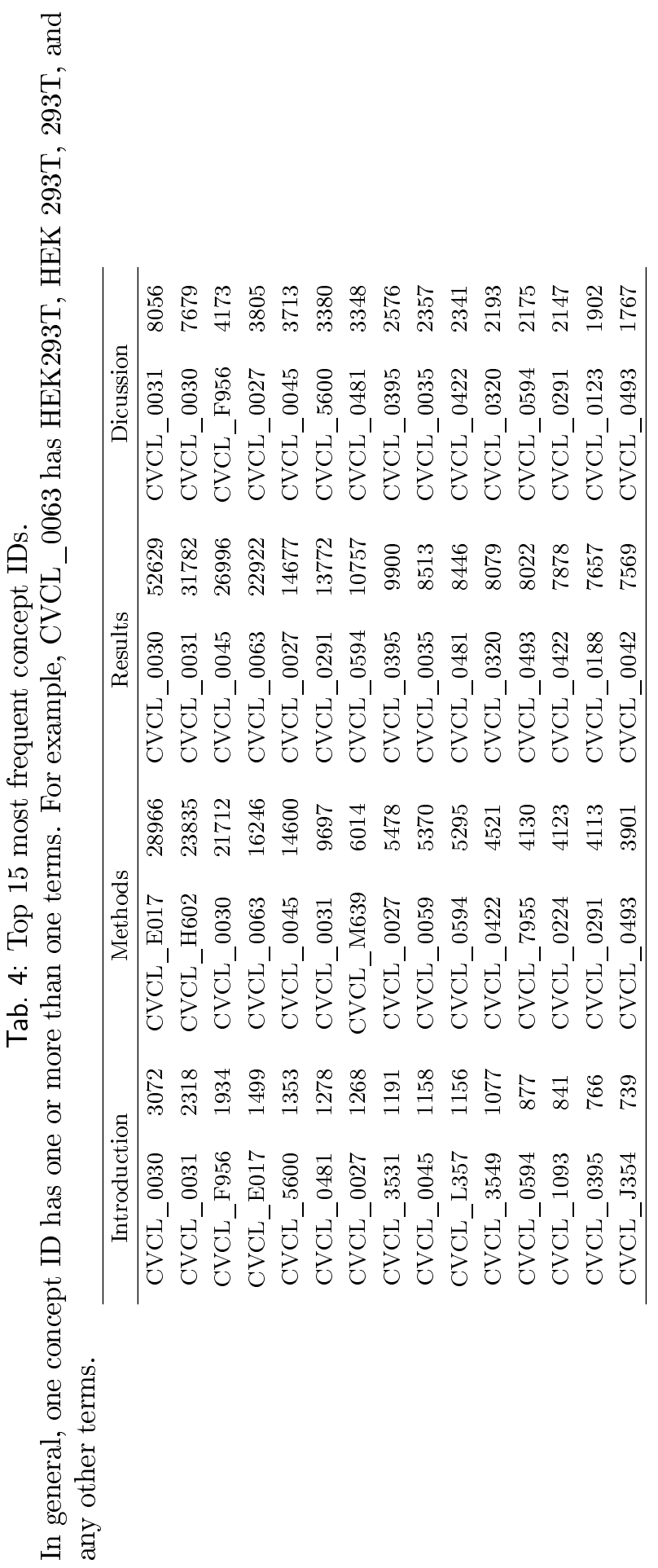




\subsection{Availability}

We annotated 633,174 Open Access (OA) articles dumped on 20 July using our cell-line tagger. Those annotated OA articles will be available on Europe PMC FTP site http://europepmc.org/ftp/oa/ner_tagging as well as REST webservice.

\section{Discussion}

In this work, we have developed a large scale annotation and analysis system for ontologies, by exploring different technologies such as semantic web, clouding computing, etc. With this system, we have annotated and provided Open Access articles annotated with Cellosaurus-based cell line tagger on $\mathrm{ftp}$ site for textmining community.

Our annotation results show that cell lines are mentioned over different sections although more often mentioned in Methods and Results sections. One surprising founding is that cell lines tagged in Results sections are less noisy then ones in Methods sections.

One application of this work is, combined with section tagger, to retrieve articles where one particular cell line mentioned in only Results sections.

As future work, we plan to extend our system adaptive and sharable using Plug and Play (P \& P) annotation concept with the following features: a simple interface, dictionary P \& P, semantic tagger P \& P, and a feature for annotation result sharing.

\section{References}

[1] Geraint Duck, Goran Nenadic, Andy Brass, David Robertson, and Robert Stevens. bionerds: exploring bioinformatics' database and software use through literature mining. BMC Bioinformatics, 14(1):194, 2013.

[2] Senay Kafkas, Jee-Hyub Kim, and Johanna R. McEntyre. Database citation in full text biomedical articles. PLoS ONE, 8(5):e63184, 052013.

[3] H. P. Luhn. A statistical approach to mechanized encoding and searching of literary information. IBM Journal of Research and Development, 1957.

[4] J. R. McEntyre, S. Ananiadou, S. Andrews, W. J. Black, R. Boulderstone, P. Buttery, D. Chaplin, S. Chevuru, N. Cobley, L.-A. Coleman, P. Davey, B. Gupta, L. Haji-Gholam, C. Hawkins, Alan Horne, S. J. Hubbard, J.-H. Kim, I. Lewin, V. Lyte, R. MacIntyre, S. Mansoor, L. Mason, J. McNaught, E. Newbold, C. Nobata, E. Ong, S. Pillai, D. Rebholz-Schuhmann, H. Rosie, R. Rowbotham, C. J. Rupp, P. Stoehr, and P. Vaughan. UKPMC: a full text article resource for the life sciences. Nucleic Acids Research.

[5] Dietrich Rebholz-Schuhmann, Miguel Arregui, Sylvain Gaudan, Harald Kirsch, and Antonio J. Yepes. Text processing through web services: Calling whatizit. Bioinformatics, pages btm557+, November 2007. 
[6] P. K. Shah, C. Perez-Iratxeta, P. Bork, and M. A. Andrade. Information extraction from full text scientific articles: where are the keywords? $B M C$ Bioinformatics, 4(1), May 2003.

[7] Nicole A. Vasilevsky, Matthew H. Brush, Holly Paddock, Laura Ponting, Shreejoy J. Tripathy, Gregory M. LaRocca, and Melissa A. Haendel. On the reproducibility of science: unique identification of research resources in the biomedical literature. 92013. 